ИЗВЕСТИЯ АКАДЕМИИ НАУК ЭСТОНСКОП ССР. ТОМ 27 ФИЗИКА - МАТЕМАТИКА. 1978, № 3

\title{
КОЛЕБАТЕЛЬНАЯ РЕЛАКСАЦИЯ МОЛЕКУЛ РbS В МАТРИЦЕ НЕОНА
}

Исследованы горячая люминесценция и внутримолекулярная колебательная релаксация молекул $\mathrm{PbS}$ в матрице неона. Приведено описание универсального криостата для спектроскопии матричной изоляции.

\section{1. Введение}

Как известно, у подавляющего большинства центров люминесценции в конденсированной среде время установления теплового равновесия по колебаниям намного короче времени жизни излучающего электронного состояния и, соответственно, вторичное свечение практически целиком сводится к обычной (термализованной) люмннесценции. Горячая люминесценция (ГЛ) $\left[{ }^{1,2}\right]$, испускаемая в ходе релаксационных процессов и тем самым позволяющая исследовать эти процессы, у таких центров обнаруживается лишь в измерениях высокой чувствительности (см. обзоры $\left.\left[{ }^{3,4}\right]\right)$. Исключительным типом центров являются некоторые легкие двухатомные молекулы (OH, OD, $\mathrm{N}_{2}$ и др.) в матрицах благородных газов $\left[{ }^{5,6}\right]$, имеющие весьма «горячие» спектры свечения, что свидетельствует о чрезвычайно больших временах внутримолекулярной колебательной релаксации, на много порядков превышающих типичные для конденсированных сред значения $\left(10^{-11}\right.$ сек). Это обусловлено слабостью взаимодействия атомов в благородной матрице и прежде всего большим числом фононов, требуемых для размена кванта весьма высокочастотного колебания легкой молекулы.

Исследования люминесценции молекул $\mathrm{PbS}$ в матрицах неона и аргона в [ $\left.{ }^{7}\right]$ показали, что спектры содержат горячие линии заметной интенсивности. Поскольку молекула $\mathrm{PbS}$ не относится к легким и частота внутримолекулярного колебания у нее на порядок меньше, чем у вышеотмеченных центров, интересно получить данные о временах жизни вибронных уровней этого центра. Это и есть одна из целей настоящей работы. Следует отметить, что такие молекулы в матрицах благородных газов актуальны с точки зрения теории колебательной релаксации как достаточно простые модельные системы: С другой стороны, матричная изоляция позволяет изучать низкотемпературные квазилинейчатые спектры широкого класса молекул, включая сложные органические соединения (см., напр., $\left.\left[{ }^{8}\right]\right)$. Чтобы решить разные задачи спектроскопии матричной изоляции, нами сконструирован криостат, описание которого приводится ниже. 


\section{2. Методика эксперимента}

Установка матричной изоляции (рис. 1 и 2) включает в себя гелиевый криостат, испарительную камеру и систему впускания матричного газа или газовой смеси в случае газообразной примеси. Криостат состоит из двух гелиевых емкостей на $1,5 \Omega(1)$ и $0,1 \Omega(2)$, соединенных с помощью двух трубок (3) и (4) из нержавеющей стали. В верхнюю гелиевую емкость помещен датчик уровня гелия (5) в виде поплавка. Терморегулирование нижней гелиевой емкости осуществляется контролируемым продуванием нижней емкости холодным газом при закрытом игольчатом вентиле $(6)$. Для заполнения нижней емкости жидким гелием вентиль (6) открывают. Откачкой гелиевых паров можно понизить температуру до $2,5 \mathrm{~K}$. Температура в нижнем сосуде поднимается выше $30 \mathrm{~K}$ с помощью намотанной на него нагревательной спирали (7). Примененный нами метод терморегулирования в криостате в принципе не отличается от метода Свенсона $\left[{ }^{9}\right]$. Датчиком температуры служит диод $(8)$ схемы Логвиненко $\left[{ }^{10}\right]$. Обе гелиевые емкости и вместе с ними объектодержатель (9) в виде медной подложки могут поворачиваться на $\pm 720^{\circ}$. Для исследования состава примесей при испарении и чистоты вакуума в криостате можно использовать вместо любого оптического окна или нижнего торца (10) корпуса криостата датчик масс-спектрометра MX-7301.

Высокотемпературная испарительная камера погружена в водяную рубашку. Через съемную заднюю стенку (11) камеры введены внутрь охлаждаемые водой токопрово́ды (12), обеспечивающие ток до $300 \mathrm{~A}$

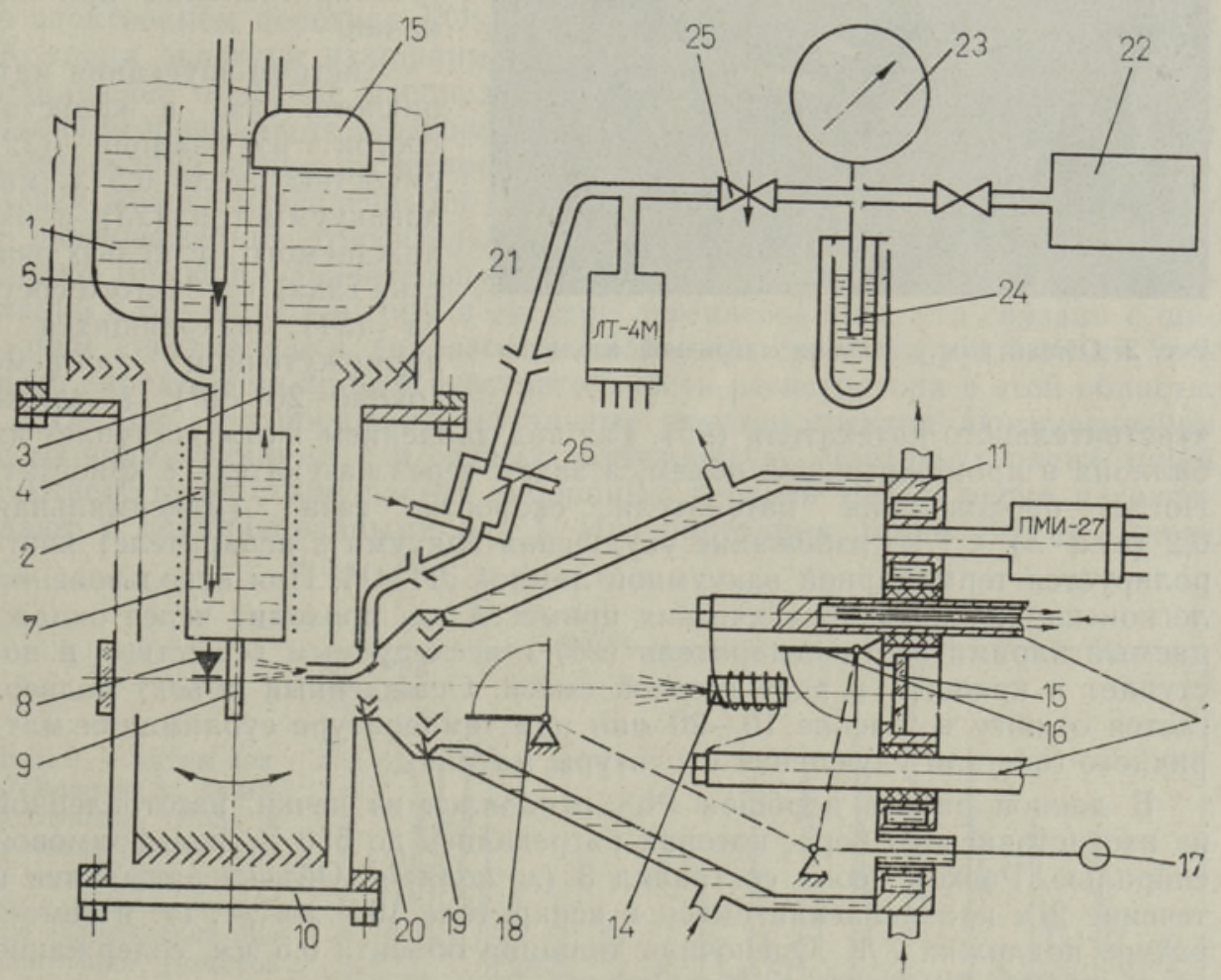

Рис. 1. Схема установки матричной изоляции. 


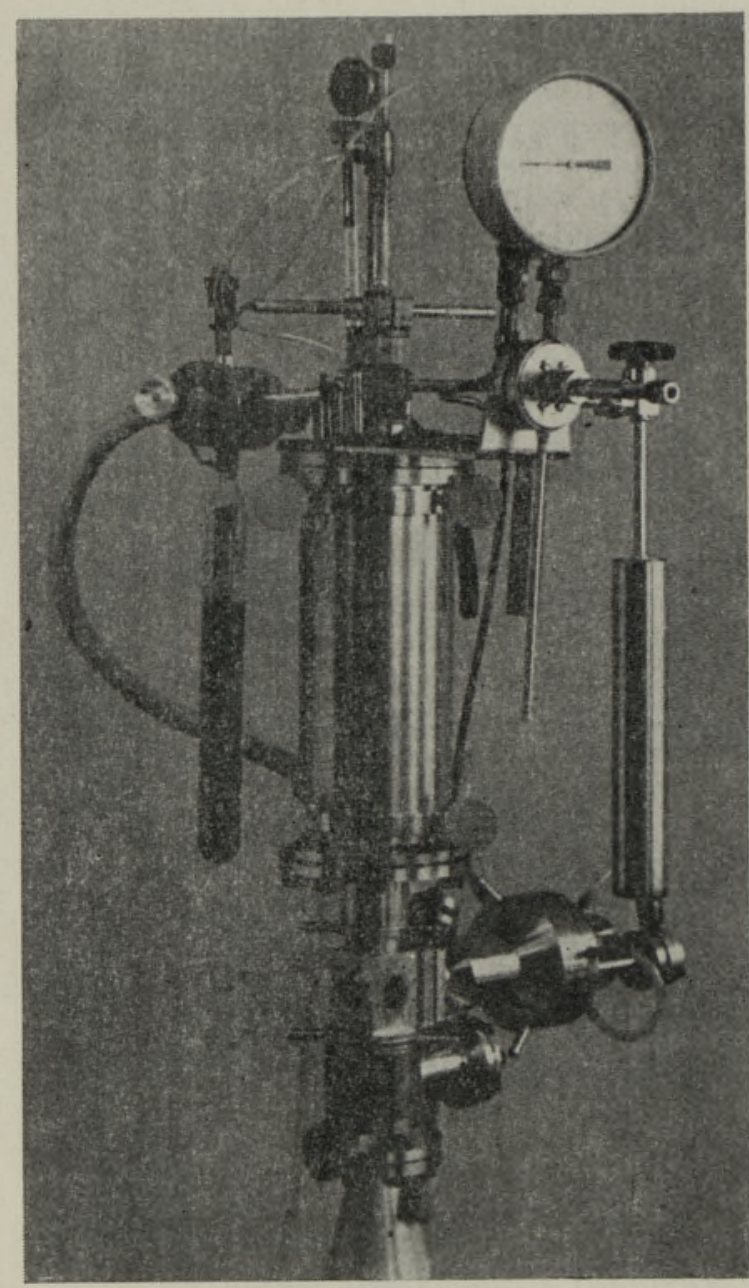

Рис. 2. Общий вид установки матричной изоляции. и температуру испарителя (14) до $3000 \mathrm{~K}$. На задней стенке имеется окно (15) для измерения пирометром температуры испарения. Ручка (17) предназначена для управления клапанами (16) и (18), первый из которых служит для защиты окна, а второй для защиты объектодержателя от загрязнения до наступления режима испарения. Высокий вакуум в испарительной камере обеспечивает не высоковакуумный насос, как в широко известных конструкциях [11], а сам криостат. Поэтому экраны (19) и (20), защищающие объект от теплового излучения, а также некоторые части экранов жидкого азота (21) изготовлены в виде жалюзи для уменьшения вакуумного сопротивления при откачке.

Система впускания матричного газа в криостат состоит из баллона (22) емкостью около $0,5 \Omega$, мановакуумметра (23) и охлаждаемого (с целью очистки газа) трубчатого сосуда (24), находящихся в промежуточном объеме (около $20 \quad c \mu^{3}$ ), а также чувствительного натекателя (25). Газ под давлением 1 атм поступает из баллона в промежуточный объем, а затем через натекатель в криостат. После прохождения натекателя скорость газа (максимальная $0,2($ атм $\cdot \Omega) \backslash \iota^{-1}$ во избежание ухудшения вакуума в испарителе) контролируется термопарной вакуумной лампой ЛТ-4М. При использовании легкоиспаряющихся органических примесей газ проходит через охлаждаемый парами азота испаритель (26) с исследуемым веществом и поступает в криостат в виде газовой смеси. Осажденный объект подвергается отжигу в течение $10-20$ мин при температуре сублимации матричного газа для улучшения структуры матрицы.

$\mathrm{B}$ данной работе порошок $\mathrm{PbS}$ испарялся из печки, изготовленной из азотистокислого бора, которая нагревалась до $550{ }^{\circ} \mathrm{C}$ вольфрамовой спиралью. Расход неона составлял $3(\mu \cdot \mu о л я) / \varkappa$. Объект напылялся в течение 2 ч при давлении смеси в испарителе $10^{-4}$ мм pт. ст. и температуре подложки $7 \mathrm{~K}$. Оценочная толщина объекта 0,5 мм, содержание молекул $\mathrm{PbS} 1: 100000$.

Возбуждение осуществлялось ксеноновой лампой ДКсШ-1000 через 
светосильный монохроматор МДР-1 или аргоновым лазером. Спектры регистрировались на спектрометрическом комплексе $\left[{ }^{12}\right]$ с автоматическим управлением и обработкой поступающих данных, сконструированном на базе монохроматора ДФС-12, ЭВМ «Наири-2», светчика фотонов ФЭУ-79 и многоканального анализатора NTA-512B.

Регистрация горячих линий велась методом многократного сканирования исследуемого участка спектра с одновременным усреднением результатов. Обработка спектров при определении параметров линий включала предварительное вычнтанне фона из-под линии.

Время затухания флуоресценции измерялось методом счета фотонов с помощью ФЭУ ЕМІ-9813В, преобразователя «время-амплитуда» «Schlumberger-7117» и многоканального анализатора NTA-1024 при возбуждении искрой между вольфрамовыми электродами в воздухе.

\section{3. Результаты}

Для определения времен релаксации по интенсивностям горячих линий необходим расчет факторов Франка-Кондона для исследуемой молекулы. С этой целью нами были исследованы спектры возбуждения разных спектральных областей свечения, проверена ндентичность этих спектров и тщательно переизмерены данные о спектре люминесценции, опубликованные в $\left[{ }^{7}\right]$. Обработанные с учетом функций чувствительности спектры показаны на рис. 3. Для распределения интенсивностей вибронных полос в спектрах выбиралось выражение [13] интегралов перекрывания гармонического осциллятора, учитывающее до второго порядка изменение частоты $\left(v\left(\mathrm{XO}^{+}\right)=430 c \mu^{-1}, v\left(\mathrm{AO}^{+}\right)=260 \mathrm{~cm}^{-1}\right)$ в электронном переходе $\mathrm{XO}^{+} \leftrightarrow \mathrm{AO}^{+}$. Учет отклонения от приближения Кондона оказался излишним. В итоге, используя машинную подгонку, наилучшее описание распределения интенсивностей достигли при значении безразмерного стоксового сдвига $z^{2}=5,3$ (ср. с номером наиболее интенсивных линий в спектрах рис. 3). Как видно из таблицы, расхождения между вычисленными и определенными из спектров возбуждения и люминесценции относительными интенсивностями линий лежат в пределах 10-20\%. Значительно меньшей $(40 \%)$ оказалась точность для линий с большим номером в спектре люминесценции, что связано с широким спектральным диапазоном вибронной серии и трудностями точного определения кривой чувствительности регистратора в этой области.

На рис. 4 изображены начальные участки спектра люминесценции с горячими линиями при разных возбуждениях. Частоты горячих линий соответствуют схеме частот вибронных уровней $\mathrm{PbS}$ в неоне и совпадают с опубликованными в $\left[{ }^{7}\right]$. Интерпретация линий ГЛ на основе

Рис. 3. Спектры возбуждения и люминесценщии $\mathrm{PbS}$ в матрице неона при $T=6$ К. Буквами $a$, 6,8 указаны частоты возбуждения при получении спектров люминесценции на рис. 4.

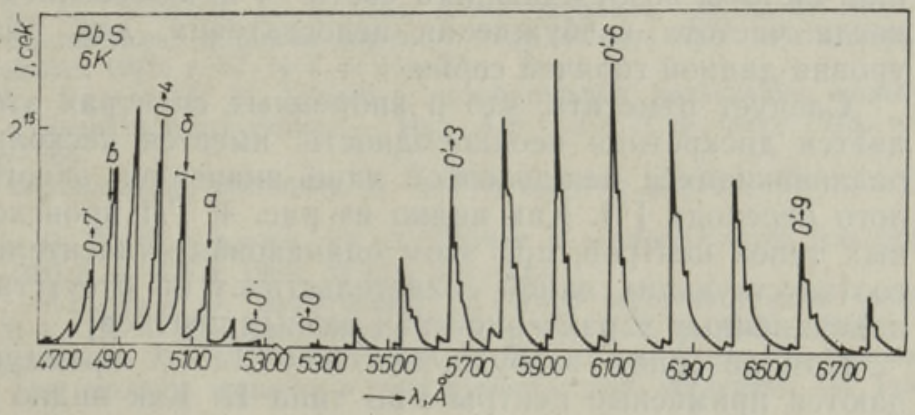


Факторы Франка-Кондона для молекулы $\mathrm{PbS}$ в переходе $\mathrm{AO}^{+} \leftrightarrow \mathrm{XO}^{+}$ Номер колебательного уровня в $\mathrm{AO}^{+}$

\begin{tabular}{|c|c|c|c|c|c|c|}
\hline & 0 & 1 & 2 & 3 & 4 & -5 \\
\hline 0 & $\frac{-}{1}$ & $\begin{array}{l}6,5 \\
6^{*}\end{array}$ & $\begin{array}{l}20 \\
18^{*}\end{array}$ & $\begin{array}{l}38 \\
34^{*}\end{array}$ & $\begin{array}{l}47 \\
45^{*}\end{array}$ & $\begin{array}{l}43 \\
47^{*}\end{array}$ \\
\hline 1 & $\begin{array}{l}4 \\
4,6^{* *}\end{array}$ & $\overline{17}$ & $\overline{30}$ & $\overline{22}$ & $\overline{4}$ & $\overline{-}$ \\
\hline 2 & $\begin{array}{l}9 \\
14^{\text {*** }}\end{array}$ & $\overline{25}$ & $\overline{20}$ & $\overline{1,6}$ & 二 & $=$ \\
\hline 3 & $\begin{array}{l}15 \\
28^{* *}\end{array}$ & $\overline{23}$ & $\overline{5}$ & 二 & $\overline{-}$ & $\overline{-}$ \\
\hline
\end{tabular}

Экспериментальные значения: * - по спектру возбуждения; ** - по спектру люминесценцин.

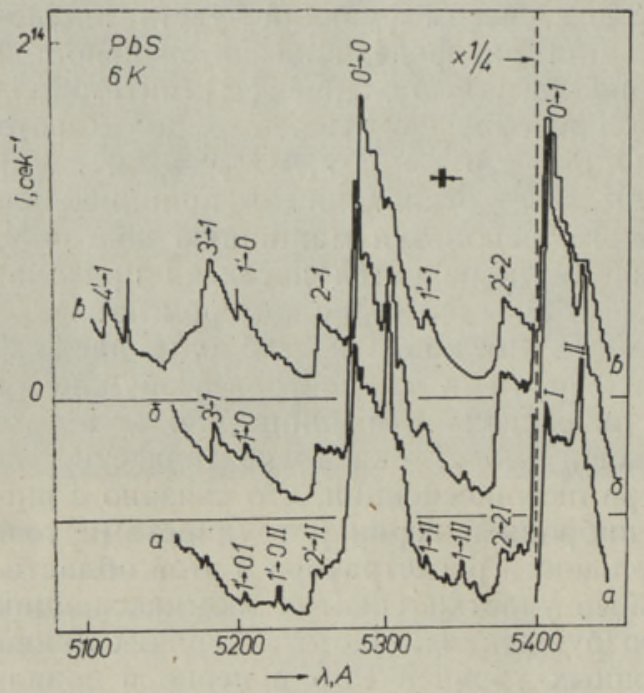

Рис. 4. Горячие линии в спектре люминесценции $\mathrm{PbS}$ в матрице неона. Буквами $a, \sigma$ и $в$ обозначены спектры при разных частотах возбуждения (см. рис. 3): $a-$ возбуждение аргоновым лазером при $\lambda=5145 \AA ; \sigma-$ ксеноновой лампой при $\lambda=5080 \AA$ с полушириной полосы $25 \AA ;$ в - аргоновым лазером при $\lambda=4880 \AA$. Буквой $\lambda$ обозначены линии газового разряда аргонового лазера и римскими цифрами линии от разных типов центров в матрице.

частотного критерия $\left[{ }^{4}\right]$ подтверждается поведением линий при изменении частоты возбуждающего света - исчезновением линий из спектра, когда частота возбуждения недостаточна для заселения исходного уровня данной горячей серии.

Следует отметить, что в вибронных спектрах этой системы наблюдается дискретная неоднородность: имеется несколько типов центров, различающихся между собой лишь значением энергии чисто электронного перехода $\left[{ }^{7}\right]$. Как видно из рис. 4 , ГЛ происходит также от разных типов центров, при этом одинаковая относительная интенсивность соответствующих линий свидетельствует об отсутствии различий в релаксационных характеристиках разных центров.

При лазерном возбуждении $\lambda=5145 \AA$ преимущественно возбуждаются примесные центры $\mathrm{PbS}$ типа II. Как видно из рис. 4, $a$, линии 
этих центров интенсивнее, чем соответствующие линии центров типа I. При остальных частотах возбуждения наблюдается люминесценция главным образом от центров типа I, так как их концентрация больше. По относительным интенсивностям горячих линий и данным таблицы определены относительные времена колебательной релаксации.

Для получения абсолютных значений времен дополнительно измерялось время затухания флуоресценции $\tau_{0}=280 \pm 20$ нсек и затем, исходя из этих данных, вычислялись времена жизни первых колебательных уровней в электронном состоянии $\mathrm{AO}^{+}$молекулы $\mathrm{PbS}$ в матрице неона $\tau_{1}, \tau_{2}, \tau_{3} \approx 2$ нсек.

Ввиду малой ннтенсивности линий ГЛ относительно фона и трудностей определения контуров линий приведенные значения могут отличаться от действительных в $1,5-2$ раза.

Итак, на примере примеси $\mathrm{PbS}$ в неоне приходим к выводу, что время колебательной релаксации у простой молекулы с умеренной частотой внутримолекулярного колебания в матрице инертного газа все же на три порядка больше, чем у подобных молекул в ионных кристаллах $\left[{ }^{2}\right]$ и у сложных органических молекул в тех же матрицах $\left[{ }^{8}\right]$. При этом относительно малая интенсивность ГЛ в случае $\mathrm{PbS}$ обусловлена в первую очередь полузапрещенным характером электронного перехода.

Авторы благодарны К. Ребане за содействие при выполнении работы и полезные замечания, Т. Соовику за помощь и советы при флуориметрических измерениях, Ә. Лухамаа за содействие при изготовлении крностата. П. Саари выражает свою признательность Ә. Никсону и Р. Текману за подробное обсуждение результатов по спектроскопии сернистого свинца $\left[{ }^{7}\right]$ и за любезное предоставление своих образцов.

\section{Л И Т Р А Т Р А}

1. Реб ан е K., С а а ри П. Время распада кванта локального колебания $\mathrm{NO}_{2}^{-}$на фононы в кристалле КСІ. - Изв. АН ЭССР, Физ. Матем., 1968, т. 17, № 2,
c. $241-244$.

2. $\mathrm{S}$ a a ri, P., Rebane, K. Hot luminescence lines in the secondary radiation spectrum of $\mathrm{KCl}-\mathrm{NO}_{2}^{-}$and $\mathrm{KBr}-\mathrm{NO}_{2}^{-}$crystals. - Solid State Comm., 1969, v. $7, \mathrm{~N} 12$, p. $887-890$.

3. Rebane, K., S a a ri, P. Hot luminescence and relaxation processes in centres of luminescence. - Proc. Internat. Conf. Luminescence, Tokyo, 1975. J. Luminescence, 1976, v. 12/13, N 1, p. 23-31.

4. Р е б а н е К. К., С а а р и П. М. Іорячая люминесценция и процессы релаксации.Изв. АН СССР, Сер. физ., 1976, т. 40, № 9, с. 1778-1784.

5. Tinti, D. S. Absorption and emission spectra of $\mathrm{OH}$ and $\mathrm{OD}$ in Solid Ne. Evidence of Radiation. - J. Chem. Phys., 1968, v. 48, N 4, p. 1459-1464.

6. T in ti, D. S., Robins o n, G. W. Spectroscopic evidence for slow vibrational and electronic relaxation in solids. The Vegord-Kaplan and second positive systems of $\mathrm{N}_{2}$ in solid rare gases. - J. Chem. Phys., 1968, v. 49, N 7, p. 32293245 .

7. Teichman, R. A., Nixon, E. R. The $A \rightarrow x$ emission spectra of $\mathrm{PbS}$ and $\mathrm{PbSe}$ in neon matrices: enhanced emission from isotopes and matrix sites. J. Mol. Spectrosc., 1975 , v. $57, \mathrm{~N} 1$, p. $14-22$.

8. С а а р и П. М., Т а м м Т. Б. Характер колебательной релаксации молекулы антрацена в матричной изоляции. - Изв. АН СССР, Сер. физ., 1978, т. 42, № 3 , c. $562-566$.

9. White, G. K. Experimental techniques in low-temperature physics. Oxford, 1968, p. 199.

10. Логвнн енко С. П., Бровкин Ю. Н. Датчик и терморегулятор для интервала $4,2-320$ К. - ПТЭ, 1968 , № 1, с. $212-213$.

11. Weltner, W., McLeod, D. Spectroscopy of carbon vapor in raregas matrices at 4 and 20 K. II. - J. Chem. Phys., 1964, v. 40, N 5, p. 1305-1316.

12. С а а и П. М., Кони Ю. Я., Пентярв Э. А., Сирк А. Э. Использование «Наири-2» как он-лайн машины в экспериментальной установке. - Тезисы 
докл. Всесоюз. науч.-техн. конф. пользователей семейства ЭВМ «Наири», Харьков, 1975 , с. $223-227$.

13. Преэм Р. А. О вычислении интегралов Франка-Кондона для гармонического осциллятора. - Тр. ИФА АН ЭССР, 1961, т. 57, № 16, с. $57-68$.

\author{
Институт физики \\ Академии наук Эстонской ССР
}

Поступила в редакцию 4/VII 1977

A. ANIJALG, A. LOHMUS, P. SAARI

\title{
PbS MOLEKULI VONKERELAKSATSIOON NEOONIMAATRIKSIS
}

Väärisgaasimaatriksites leidub erandlikult aeglase võnkerelaksatsiooniga molekule, mille luminestsents sisaldab oluliselt kuuma komponenti. Et hiljuti on leitud märgatava intensiivsusega kuumi jooni ka raske molekuli $\mathrm{PbS}$ spektrist $\left[{ }^{7}\right]$, osutus huvitavaks uurida selle tsentri võnkerelaksatsiooni kiirust. Selleks mõōdeti PbS molekuli ergastus- ja luminestsentsispektreid neoonis temperatuuril $6 \mathrm{~K}$, määrati Stokes'i kadude parameeter $z^{2}=5,3$ kvanti - ning mōõdeti kiirgava elektronseisundi eluiga $-\tau_{0}=280$ ns. Peale selle määrati $\mathrm{PbS}$ molekuli ergastatud elektronseisundi $\mathrm{AO}^{+}$esimeste võnkeseisundite eluead $\tau_{1}, \tau_{2}, \tau_{3}$, mille väärtuseks saadi ca 2 ns.

Süsteemi vōnkerelaksatsioon on seega küll kiirem kui kergete molekulide puhul, kuid siiski kolm suurusjärku aeglasem kui molekulaartsentritel ioonsetes kristallides vôi orgaanilistel molekulidel väärisgaasides; kuuma luminestsentsi märgatav osa on tingitud elektronülemineku keelatud iseloomust. Töös on kirjeldatud universaalset krüostaati, mis võimaldab mitmesugustes gaasimaatriksites spektroskoopiliselt uurida nii orgaanilisi kui ka anorgaanilisi molekule, mille sublimatsioonitemperatuur on vahemikus 300$3000 \mathrm{~K}$.

\section{A. ANIJALG, A. LOHMUS, P. SAARI}

\section{VIBRATIONAL RELAXATION OF PbS MOLECULE IN NEON MATRIX}

It has been known that a number of diatomics with exceptionally slow relaxation in rare gas matrices show a considerable hot component in the luminescence spectra. Recently hot lines of marked intensity were observed also in the spectrum of heavy diatomic $\mathrm{PbS}\left[{ }^{7}\right]$; therefore it was of interest to clear up the relaxation rate of this centre. For this purpose the luminescence and excitation spectra of $\mathrm{PbS}$ in neon were measured at $6 \mathrm{~K}$ and the parameter of Stokes losses $z^{2}=5.3$ quanta was determined; the lifetime of the electronic state $\mathrm{AO}+\tau_{0}=$ $=280 \pm 20 \mathrm{~ns}$, was also measured. As a result the lifetimes of the first excited vibrational levels in the electronic state $\mathrm{AO}+\tau_{1} \approx \tau_{2} \approx \tau_{3} \approx 2 \mathrm{~ns}$, were evaluated. Hence the vibrational relaxation in the system studied is fast if compared with light diatomics, but up to three orders of magnitude slower than that of the impurity centres in alkali halides or of the organic molecules in rare gas matrices and, consequently, the rather weak intensity of hot luminescence is due to the semiforbidden nature of electronic transition. A multi-purpose cryostat, which allows to perform spectroscopic studies of anorganic as well as organic molecules with sublimation temperature from 300 to $3000 \mathrm{~K}$ in various gas matrices, is also described in this paper. 